\title{
Experience of Unilateral Cleft Lip Repair using the Anatomical Subunit Technique in an Omani Population over a Five-Year Period
}

"Sheikhan Al Hashmi, ${ }^{1}$ Malak Al Wahaibi, ${ }^{2}$ Sony P. Varghese, ${ }^{1}$ Maather Al Abri, ${ }^{3}$ Moath A. Shummo, ${ }^{1}$ Buthina Al Muqbali ${ }^{1}$

\begin{abstract}
Objectives: This study aimed to report the authors' experience with the anatomical subunit technique for unilateral cleft lip repair, which has gained popularity worldwide. Methods: From July 2015 to April 2020, 114 consecutive cases of cleft lip underwent primary cleft lip repair with closed rhinoplasty by a single surgeon. The demographic data, severity and type of the cleft lip, surgical outcomes, including vermillion notching, were assessed by an independent senior surgeon. The rate of revision surgery was collected from the Al-Shifa ${ }^{\circledR}$ - 3Plus healthcare information system (Ministry of Health, Oman). Parents' satisfaction regarding scar quality and lip and nose appearance was collected and all data were statistically analysed. Results: A total of 82 cases satisfied the inclusion criteria. The mean age at surgery for cleft lip was 32 weeks. Among these, 35 cases (43\%) were complete cleft lips and 47 cases (57\%) were incomplete. Additionally, 43 children (52\%) were born of consanguineous marriage. Six patients (7\%) needed revision surgery. The digital survey was completed by 40 subjects (response rate: $48.8 \%$ ) which showed $85 \%$ satisfaction rate with the postoperative scar and $77.5 \%$ satisfaction with the aesthetic appearance of the nose. Conclusion: The anatomical subunit technique resulted in a predictable outcome indicating a high rate of patient satisfaction with scar quality and nasal and lip symmetry in children with varying severity of cleft lip. The high percentage of consanguinity (52\%) in this study highlights the need for more targeted national campaigns involving premarital counselling in the Omani population.
\end{abstract}

Keywords: Cleft Lips; Congenital Abnormalities; Cleft lip/Surgery; Reconstructive Surgical Procedures; Treatment Outcome; Surgical Diagnostic Technique; Consanguinity; Oman.

\section{Advances IN KNOWLedge \\ To the best of the authors' knowledge, this is the first study in Oman to highlight the outcomes and direct experience of this particular technique for cleft lip repair in an Omani population. \\ This study revealed a high percentage of consanguinity (52\%) in the study group. \\ The most common complication associated with the anatomical subunit technique in the current study was vermilion excess, which was corrected with simple wedge excision. \\ There was an $85 \%$ satisfaction rate with the postoperative scar and $77.5 \%$ satisfaction rate with the aesthetic appearance of the nose.}

\section{Application to Patient Care}

The anatomic subunit technique used here produced predictable and identical results related to scar quality and nasal and lip symmetry in children with varying severity of cleft lip.

Due to the high consanguinity in this study group, the authors are calling for more targeted national campaigns involving premarital counselling in the Omani population.

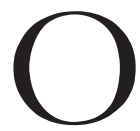

ROFACIAL CLEFTS REMAIN THE MOST common craniofacial congenital anomaly. ${ }^{1,2}$ A study published in 2001 by Rajab and Thomas concluded that the prevalence of cleft lip in Oman was 15 per 10,000 births. $^{3}$ Out of those, 0.62 per 1000 live births were combined cleft lip and palate and 0.34 per 1000 live births were isolated cleft lip cases. ${ }^{3}$ This result is striking as it suggests a higher prevalence of cleft lip in Oman in relation to worldwide numbers. The Data published from the International Perinatal Database of Typical Oral Clefts in 2011 stated that the worldwide prevalence of cleft lip was found to be 9.9 per 10,000 births, compared to 15 per 10,000 births in
Oman. ${ }^{4}$ The incidence of cleft lip has been found to be associated with consanguineous marriages, specifically in children born to parents who are first cousins which was found to be $61.1 \%$; this incidence falls down to $18.0 \%$ in parents who are second cousins. ${ }^{5}$ A metaanalysis of orofacial clefts indicated a clear relationship between consanguinity and non-syndromic orofacial clefts (NSOFC), with the risk being twice in children born to consanguineous parents. ${ }^{5}$

Cleft lip anomalies are more commonly found in association with cleft palate deformities; however, in other less common instances, it can present as an isolated cleft lip anomaly. ${ }^{1,6}$ Cleft lip anomalies can be 
unilateral or bilateral and can range in severity from a scale of a microform cleft lip which is considered the least disfiguring, to a complete cleft lip., ${ }^{1,7}$ Some of the well-known challenges of children born with cleft lip include feeding difficulties, trouble in articulation and speech development along with psychological and self-esteem concerns related to the condition. ${ }^{1}$ Studies related to the comprehensive statistics of this anomaly and its management outcomes in Oman remain significantly limited.

Different studies have reported their experience with the anatomical subunit technique in comparison to Millard and Modified Millard technique. Deshmukh et al. published a study favouring the aesthetic outcomes of Fisher's technique over Mohler's technique. ${ }^{8}$ In the study, 50 patients were selected and randomly assigned to undergo surgical cleft lip repair by either the Mohler or Fisher technique performed by a single surgeon. Outcomes were then assessed by laymen and demonstrated that a bigger proportion of evaluators have favoured the aesthetic outcomes of the Fisher repair. ${ }^{8}$

Kwang et al. also published a similar study, which enrolled candidates from different levels of training and requested them to evaluate the aesthetic outcomes of anatomical subunit repair in comparison to rotation advancement repair. ${ }^{9}$ In this study, candidates from different levels of training have found outcomes of the anatomical subunit techniques to be aesthetically superior to that of the latter technique. ${ }^{9}$ The experience of the anatomic subunit technique has not been studied in the Omani population prior to this study.

Given that the anatomical subunit technique for unilateral cleft lip repair has gained popularity worldwide, this study aimed to report the present authors' experience with the aforementioned technique. A related aim was to explore the opinion and experience of parents whose children had undergone cleft lip repair using the above technique and provide a clearer picture of the demographic data in a population of Omani patients with cleft lip.

\section{Methods}

This is a retrospective study of the surgical correction of the cleft lip anomaly in an Omani population. The procedure was performed using the anatomic subunit technique of cleft lip repair by single surgeon over a period of 5 years - July 2015 to April 2020-for varying degrees of cleft lip ranging from unilateral incomplete cleft lip to complete cleft lip. The institute indicated in this study, Khoula hospital, is a level 1 trauma centre and is also the main plastic surgery centre in Oman.
The standard technique used for the correction of cleft lip in this institute is the Millard or modified Millard Rotation Advancement Technique. The anatomic subunit technique was introduced to the department of plastic surgery by one of the authors in 2015 .

All children who underwent cleft lip repair from July 2015 to April 2020 using the anatomic subunit technique of cleft lip repair at Khoula hospital, Muscat, Oman were included in this study. The records were collected using the Al-Shifa ${ }^{\circledR}$ 3Plus healthcare information system, (Ministry of Health, Oman). Children who did not follow up, parents who refused to be part of the study due to social or cultural reasons, children with a microform cleft lip, children with bilateral cleft lip, or patients with inadequate data entered into the above-mentioned e-healthcare system were all excluded.

All patients were evaluated preoperatively in the clinic and prioritised according to the age and anaesthetic clearance for surgery. Nasoalveolar moulding by an experienced orthodontist was requested for indicated cases. Most of the children were operated on by the fifth to seventh month of age after adequate weight gain and haemoglobin evaluation. Once the children were cleared by the pre-anaesthetic clinic for surgery, they were admitted and operated on the following day. All the children underwent cheiloplasty using the anatomic subunit technique with closed rhinoplasty by a single surgeon under general anaesthesia.

The procedure started with markings as per the anatomical subunit technique following which the key landmarks were tattooed. Infiltration of xylocaine $1 \%$ with 1:200,000 adrenaline in the alar base and inferior turbinate area on the cleft side was performed. Incisions were made on the medial lip along the markings including the opening triangle. A cuff of the orbicularis oris muscle was dissected after releasing its attachments to the columella base and alveolar cleft margin. Following this, lateral lip incisions were made along the markings and after discarding the tissue along the cleft margin, a cuff of the orbicularis oris muscle was dissected in the same manner. The alar base on the cleft side was then released off the pyriform and advanced anteromedially. The closure started with the nasal floor and, in some cases, a turbinate flap was used to cover any exposed periosteal surface of the pyriform margin. Primary closed rhinoplasty was done with repositioning of the cleft side dome using 5-0 Monocryl (Ethicon Inc., Cornelia, Georgia, USA). The mucosa, the muscle and the lateral vermilion flap were each approximated with 5-0 Vicryl (Ethicon Inc., Johnson \& Johnson, Somerville, New Jersey, USA) and the skin is closed with 7-0 Prolene 

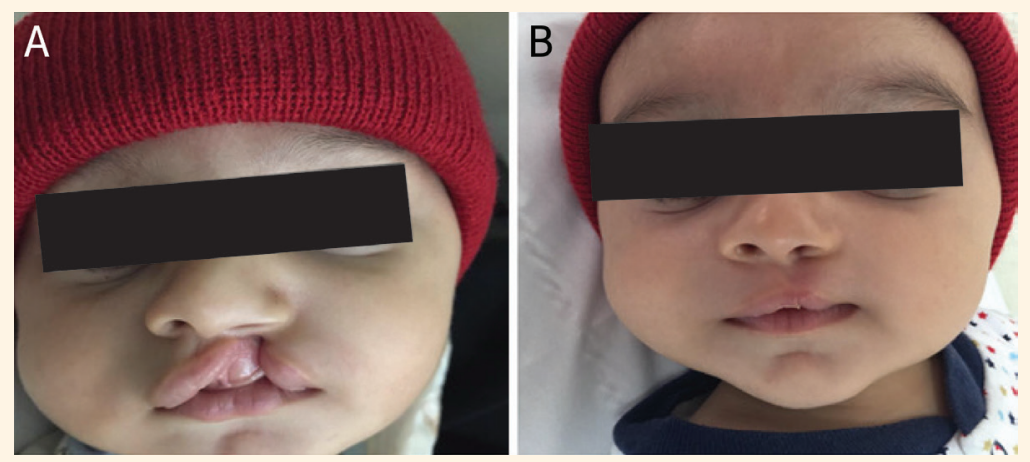

Figure 1: A pre-operative image of an infant (A) showing left-sided incomplete cleft lip and post-operative image (B) showing left-sided incomplete cleft lip following surgery using the anatomical subunit technique.

(Ethicon, Inc., Manlo Park, California, USA). An intraoperative nostril retainer was applied in all cases and secured using a 4/0 nylon monofilament suture to the membranous septum. Elbow splints were applied and maintained for 10-14 days. The children were allowed to be breast fed 3-4 hours post-operation and discharged on the first or second postoperative day. They were re-evaluated at three weeks, six weeks, three months, six months and then annually at the clinic. The average long-term follow-up for patients in this study was 2.3 years. The postoperative evaluation of all children who underwent cleft lip repair included evaluation and assessment of the quality of the scar on the lip and vermilion, symmetry of the nose, symmetry of the vermilion volume and white roll alignment by an independent senior surgeon.

Parents of all the children who had undergone cleft lip repair using the anatomic subunit technique of cleft lip repair at the hospital were sent a link to an electronic survey regarding the aesthetic appearance of the lip after surgery, ability to breast feed and their satisfaction with surgical scar and with the correction of the nasal deformity. All other data required for the study was collected from the Al-Shifa ${ }^{\circledR}$ 3Plus healthcare information system (Ministry of Health, Oman). This included personal data, date of surgery, severity and side of cleft lip, presence of a protruding premaxilla, pre-surgical orthodontics, grade of cleft palate, associated syndromes, the geographical area the child is from, associated anomalies, consanguinity, family history of cleft, last follow-up date, objective assessment and any revision surgery for the lip. The data were entered into excel sheets and analysed using Statistical Package for the Social Sciences (SPSS), Version 24.0 (IBM Corp., Chicago, Illinois, USA).

Ethical approval was obtained from the Ministry of Health, Centre of Studies and Research at Oman and the Research Ethics Committee at Khoula Hospital (Unique Identification code: 24273) Verbal consent was obtained from the parents who were interested in filling out the electronic survey and for those willing to include their children's photos in this article.

\section{Results}

A total of 114 cleft lip surgeries were performed by one of the authors during the study period. Out of 114 patients, 82 satisfied the inclusion criteria and were included in the study. Out of the included cases, 52 were males (63\%) and 30 were females (37\%). The mean age of the patients at the time of surgery was 32 weeks. Nine children (11\%) underwent the surgery in following weeks due to late presentation and comorbid conditions. The eldest of the patients presented for the first time at 356 weeks due to personal and socioeconomic issues and was operated on at the age of presentation. A total of $43 \%$ of the cleft lip cases were from the Muscat and A'Dakhiliya governorates with 18 (22\%) and 17 (21\%) cases, respectively. The other regions made up $57 \%$ of the cases with A'Sharqiah, $\mathrm{Al}$ Batinah, A'Dhahirah, Al Wusta, Musandam and Al Buraimi representing 20\%, 19\%, 11\%, 4\%, 2\% and 1\% of the cases, respectively. All cleft lip surgeries were conducted using the anatomical subunit technique.

It was found that 35 cases (43\%) presented with complete cleft lip and 47 cases (57\%) were found to be incomplete. Additionally, 53 cases (65\%) were left-sided and 29 cases (35\%) were right-sided. It was also noted that 36 patients (44\%) had cleft of palate involving both hard and soft palate, out of which 28 patients (34\%) qualified for Veau's criteria of class III cleft palate and eight patients (10\%) qualified for class II. Additionally, 21 patients (26\%) underwent presurgical nasoalveolar moulding and three patients had protruding premaxilla; 12 patients had associated anomalies which were mainly cardiac anomalies. Two children were confirmed to be syndromic-one had Downs syndrome and the other, velocardiofacial syndrome. Additionally, 43 children (52\%) were born of 

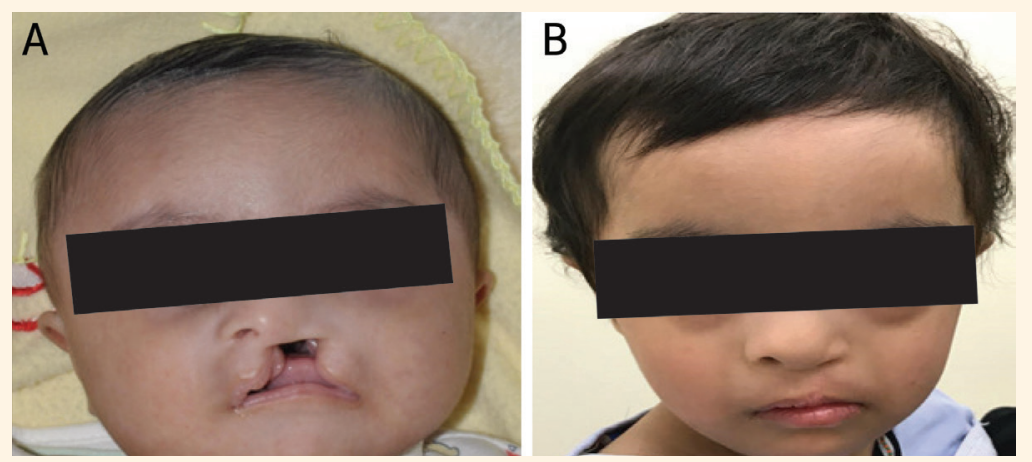

Figure 2: A pre-operative image of a child (A) showing left-sided complete cleft lip and post-operative image (B) showing left-sided complete cleft lip following surgery using the anatomical subunit technique.

consanguineous marriages: 33 (77\%) from a first cousin marriage, 7 (16\%) from a second cousin marriage and 3 (7\%) from a third cousin marriage. It was found that 22 patients (27\%) had a positive family history of cleft lip. Out of the 82 who had undergone cleft lip repair using the anatomic subunit technique, six patients (7\%) needed revision as judged by the senior independent surgeon at the time of the last follow-up during the study period, five of which were for vermilion excess, managed through simple wedge resection. Collectively, the repair was performed on both complete and incomplete cleft lips and both produced identical results [Figures 1 and 2]. A digital survey was sent to the parents of the 82 children who had undergone cleft lip repair using the anatomical subunit technique. Out of these, the parents of 40 children responded to the survey anonymously (response rate: $48.8 \%)$. Statistical analysis showed that $82.5 \%$ (33/40) were satisfied with the aesthetic appearance of lip following cleft lip repair by the anatomical subunit technique, 85\% (34/40) were satisfied with the postoperative scar over the lip and $77.5 \%(31 / 40)$ were satisfied with the aesthetic appearance of nose following cleft lip surgery with closed rhinoplasty.

\section{Discussion}

Various techniques have been used by plastic surgeons to correct cleft lip anomalies, all of which have aimed to establish the best functional rehabilitation and aesthetical acceptance. ${ }^{10-13}$ Rose's straight-line repair was one of the first cleft lip repair techniques to be described; this technique was published in 1891, followed by the rotation advancement technique for unilateral cleft lip repair introduced by Ralph Millard in 1957. This technique is initiated by forming an incision just below the nostril which continues to run vertically to the philtral ridge. ${ }^{12}$ Millard's technique has undergone a number of refinements throughout the years but probably remains the most common technique used in unilateral cleft lip repair worldwide. ${ }^{13}$
Mohler further modified the rotation-advancement repair technique in 1987, achieving a more symmetric positioning of the scar. The next big evolution in cleft lip repair came with the introduction of the extended Mohler cleft lip repair by Court Cutting in 2003. ${ }^{14}$ The major adjustment of this technique was to extend the incision to the noncleft philtral column. ${ }^{11}$ With this modification, two major shortcomings arose: first was the complex appearance of the scar under the nasal sill, which did not blend with the anatomical landmarks; the second was that in order to avoid the under rotation and elevation of the cupid's bow peak, the lateral lip segment was shortened. ${ }^{11}$

To address the aforementioned shortcomings, David M. Fisher proposed the anatomic subunit technique first published in 2005. ${ }^{11}$ As the name suggests, this technique respects the anatomical subunits of the lip and is based on accurate preoperative measurements. This technique preserved the transverse length of the lateral lip by creating a cutaneous triangle flap above the white roll to correct the height of the medial lip. ${ }^{11}$ Ever since this technique had been introduced, the anatomical subunit technique gained notable popularity. ${ }^{7}$ A recent study conducted in 2020 aimed to explore the current practice patterns in unilateral cleft lip repair among surgeons within the American Cleft Palate Association. It concluded that up to $40 \%$ of surgeons had changed their previously implemented techniques of cleft lip repair to the anatomical subunit approximation technique. ${ }^{7}$ Fisher's technique of cleft lip repair has also been found to have a lesser revision rate for lip shortening, scar hypertrophy and scar widening in comparison to the rotation advancement technique. ${ }^{15}$ However, Fisher's technique was found to be associated with a higher number of vermilion revisions which can be managed easily by a simple wedge excision of the vermilion.

As mentioned above, Millard's technique for cleft lip repair remains one of the most implemented and taught methods for correcting the cleft lip anomaly worldwide. ${ }^{16}$ This has also been the case in the present 
institution. The anatomical subunit technique was introduced in the department by one of the authors in 2015 , and since then, it has been gaining popularity. The experience of this technique has not been studied in the Omani population prior to this study.

Oman is a country nestled in the tip of Arabian Peninsula with a population of 4.5 million and land area of $309,500 \mathrm{~km}^{2}$ encompassing 11 governorates. ${ }^{17}$ As per certain traditional values preserved within this rapidly modernising country, consanguineous marriages appear to be prevalent in the local population. Local research has indicated that more than half (52\%) of marriages are consanguineous, with first cousin unions being the most common type of consanguineous marriages constituting of $39 \%$ of all marriages and $75 \%$ of consanguineous marriages. ${ }^{3}$ Owing to this cultural practice of consanguineous marriages in Oman, the incidence of clefts has been found to be almost 1.5 times the worldwide prevalence. ${ }^{3}$

In the authors' experience, the outcome of cleft lip repair using the anatomical subunit technique has been very satisfactory, a fact that can be corroborated by the small number of revision surgeries required by the patients involved in the current study. Excess vermillion is a recognised outcome of the anatomical subunit technique. For example, a study published by Mittermiller et al. revealed that $37 \%$ of patients in their study group who underwent unilateral cleft lip repair using the technique required debulking of excess vermillion. ${ }^{18}$ Furthermore, the parents' satisfaction rate following the surgery in this study was $82.5 \%$. Most of the parents were well-accepting of the surgical repair with anatomical subunit technique and willing to recommend the procedure to other children with similar anomalies. Similarly, a study by Deshmukh et al. has aimed to compare the post-operative aesthetic outcomes between Fisher's and Mohler's technique. ${ }^{8}$ The comparison was done through a layman evaluation of the post-operative cases based on which the authors were able to confirm the superior aesthetic outcomes of Fisher's technique. ${ }^{8}$

Another factor of comparison with the international literature was the implementation of Fisher's technique on a different spectrum of cleft lip severity. Out of the 82 patients included in our study, 36 had varying clefts of the soft and hard palate. No significant difference was found in the outcome of cleft lip repair done in children with isolated cleft lip in comparison to children with combined cleft lip and palate. This finding was also true with other published studies, indicating that with accurate application of the technique, the outcomes will less likely be affected by the severity of the cleft lip..$^{15,16,19}$
The high percentage of consanguinity (52\%) in the current study group highlights the need for targeted national campaigns involving premarital counselling regarding consanguinity, especially as this study revealed that consanguineous families are more likely to have a first degree relative with cleft lip. Out of the 82 children, two were confirmed to be syndromic, one was a case of trisomy 21 and the other was diagnosed with velocardiofacial syndrome. The current syndrome-related findings are also similar to the patterns found internationally, indicating velocardiofacial syndrome along with other previously mentioned syndromes to be commonly associated with this anomaly. ${ }^{20}$ The most common complication associated with anatomical subunit technique was vermilion excess which were corrected for six patients in this study using simple wedge excision done 18-24 months after the initial lip surgery.

The limitation of the current study includes the absence of a comparison group. Comparison of the present cases with cases operated on using another technique would have provided better insight into the superiority of the anatomical subunit technique. In addition, a more accurate temporal relationship might have been established if the study was prospective in nature. Lastly, in order to obtain more transparent results, the participation of families in the electronic survey was voluntary and anonymous. However, voluntary participation commonly leads to lower response rates and some families have refused to participate in the survey for various socio-cultural reasons.

\section{Conclusion}

To the best of the authors' knowledge, this is the first study in Oman to highlight the outcomes and patients' experience of the anatomical subunit technique for cleft lip anomaly in an Omani population. Accordingly, it resulted in predictable and identical results related to scar quality and nasal and lip symmetry in children with varying severity of cleft lip. Revision for vermilion excess was needed in 7\% of the cases. The high percentage of consanguinity in this study highlighted the need for more targeted national campaigns involving premarital counselling in the Omani population as consanguineal marriages have been commonly implicated in the development of the cleft lip anomaly.

\section{AUTHORS' CONTRIBUTION}

SH conceptualised and designed the study as well as supervised the work. MW, SPV, MA and MAS 
collected the data. BM was responsible for patient coordination in this study. MA analysed the data. MW and SPV drafted the manuscript. All authors approved the final version of the manuscript.

\section{CONFLICT OF INTEREST}

The authors declare no conflicts of interest.

\section{FUNDING}

No funding was received for this study.

\section{References}

1. Trainor PA. Craniofacial birth defects: The role of neural crest cells in the etiology and pathogenesis of Treacher Collins syndrome and the potential for prevention. Am J Med Genet A 2010; 152A:2984-94. https://doi.org/10.1002/ajmg.a.33454.

2. Kantar RS, Cammarata MJ, Rifkin WI, Plana NM, Diaz-Siso JR, Flores RL. Outpatient versus inpatient primary cleft lip and palate surgery: Analysis of early complications. Plast Reconstr Surg 2018; 141:697e-706e. https://doi.org/10.1097/PRS.00000 00000004293 .

3. Rajab A, Thomas C. Oral clefts in the Sultanate of Oman. Eur J Plast Surg 2001; 24:230-3. https://doi.org/10.1007/ s002380100253.

4. IPDTOC Working Group. Prevalence at birth of cleft lip with or without cleft palate: data from the International Perinatal Database of Typical Oral Clefts (IPDTOC). Cleft Palate Craniofac J 2011; 48:66-81. https://doi.org/10.1597/09-217.

5. Sabbagh HI, Innes NP, Sallout BI, Alamoudi NM, Hamdan MA, Alhamlan N, et al. Birth prevalence of non-syndromic orofacial clefts in Saudi Arabia and the effects of parental consanguinity. Saudi Med J 2015; 36:1076-83. https://doi.org/10.15537/ smj.2015.9.11823.

6. Sisti A, Nisi G. Principles of cleft lip repair: Conventions, commonalities, and controversies. Plast Reconstr Surg 2017; 140:833e-4e. https://doi.org/10.1097/PRS.0000000000003884.

7. Roberts JM, Jacobs A, Morrow B, Hauck R, Samson TD. Current trends in unilateral cleft lip care: A 10-year update on practice patterns. Ann Plast Surg 2020; 84:595-601. https://doi. org/10.1097/SAP.0000000000002017.
8. Deshmukh M, Vaidya S, Deshpande G, Galinde J, Natarajan S. Comparative evaluation of esthetic outcomes in unilateral cleft lip repair between the Mohler and Fisher repair techniques: A prospective, randomized, observer-blind study. J Oral Maxillofac Surg 2019; 77:182.e1-182.e8. https://doi. org/10.1016/j.joms.2018.08.029.

9. Kwong JW, Cai LZ, Azad AD, Lorenz HP, Khosla RK, Lee GK, et al. Assessing the Fisher, Mohler, and Millard techniques of cleft lip repair surgery with eye-tracking technology. Ann Plast Surg 2019; 82:S313-19. https://doi.org/10.1097/ sap.0000000000001911.

10. Wehby GL, Cassell CH. The impact of orofacial clefts on quality of life and healthcare use and costs. Oral Dis 2010; 16:3-10. https://doi.org/10.1111/j.1601-0825.2009.01588.x.

11. Kim HY, Park J, Chang MC, Song IS, Seo BM. Modified Fisher method for unilateral cleft lip-report of cases. Maxillofac Plast Reconstr Surg 2017; 39:12. https://doi.org/10.1186/s40902017-0109-1.

12. Monson LA, Kirschner RE, Losee JE. Primary repair of cleft lip and nasal deformity. Plast Reconstr Surg 2013; 132:1040e-53e. https://doi.org/10.1097/prs.0b013e3182a808e6

13. Knežević P, Vuletić M, Blivajs I, Dediol E, Macan D, Virag M. The modification of rotation - Advancement flap made in 1950. Acta Stomatol Croat 2017; 51:60-4. https://doi.org/10.15644/ $\operatorname{asc} 51 / 1 / 8$

14. Mohler LR. Unilateral cleft lip repair. Plast Reconstr Surg 1987; 80:511-17. https://doi.org/10.1097/00006534-198710000-00005.

15. Ajmal S, Khan MA, Khan AT, Yousaf K, Shadman M, Iqbal T. Evaluating anatomical subunit approximation technique for unilateral cleft repair. J of Postgrad Med Inst 2010; 24:68-72.

16. Tse R, Lien S. Unilateral cleft lip repair using the anatomical subunit approximation: Modifications and analysis of early results in 100 consecutive cases. Plast Reconstr Surg 2015; 136:119-30. https://doi.org/10.1097/prs.0000000000001369.

17. Foreign Ministry of Oman. Geography. From: https://fm.gov. om/about-oman/state/geography/ Accessed: Jun 21.

18. Mittermiller PA, Martin S, Johns DN, Perrault D, Jablonka EM, Khosla RK. Improvements in cleft lip aesthetics with the Fisher repair compared to the Mohler repair. Plast Reconstr Surg Glob Open 2020; 8:e2919. https://doi.org/10.1097/GOX.00000 00000002919.

19. Fisher DM. Unilateral cleft lip repair: An anatomical subunit approximation technique. Plast Reconstr Surg 2005; 116:61-71. https://doi.org/10.1097/01.prs.0000169693.87591.9b.

20. Venkatesh R. Syndromes and anomalies associated with cleft. Indian J Plast Surg 2009; 42:S51-5. https://doi.org/10.41 03/0970-0358.57187 